\title{
Relationship of left atrial size and function to invasive left ventricular filling pressure: a cardiac MRI study
}

\author{
Kanna Posina*, Jeannette McLaughlin, Peter D Rhee, George A Petrossian, Nathaniel Reichek, Jie J Cao \\ From 15th Annual SCMR Scientific Sessions \\ Orlando, FL, USA. 2-5 February 2012
}

\section{Background}

Increased left atrial (LA) size measured at left ventricular (LV) end systole is associated with cardiovascular morbidity and mortality in population based studies. LA function can be divided into 3 components: LA filling during LV systole, a passive emptying phase during early diastole and active atrial systole during LV late diastole. We sought to assess the relationships of LA volume and emptying function to invasive LV end diastolic pressure (LVEDP).

\section{Methods}

Forty one patients underwent cardiac MRI (CMR) within 5 hours of clinically indicated left heart catheterization. Sixteen healthy volunteers served as CMR controls. LV and LA volumes and ejection (EF) were assessed from cine images. Using the biplane formula, LA volume was calculated at LV end-systole (LAVmax), LV end-diastole (LAVmin) and at the end of LV early filling before LA systole. LA function was divided into global emptying function (LAEF), passive LAEF and active LAEF expressed as fractional volume change. LVEDP was measured invasively during diagnostic catheterization. The association of LA indices with LVEDP was assessed using Receiver Operating Characteristics.

\section{Results}

Mean age was 59 years in patients and 53 years in control (NS). As compared to controls, patients had larger LVEDV $(80 \pm 15 \mathrm{ml} / \mathrm{m} 2$ vs. $90 \pm 39 \mathrm{ml} / \mathrm{m} 2, \mathrm{p}=0.029)$ and lower LVEF $(57 \pm 5 \%$ vs. $49 \pm 16 \%, p=0.006)$. Mean LVEDP was $14 \pm 8 \mathrm{mmHg}$ in patients. Of the LA volume indices LAVmin was significantly more closely associated with LVEDP elevation ( $>12 \mathrm{mmHg}$ ) (AUC 0.765, $\mathrm{p}=0.002)$ than LAVmax (AUC 0.677, $\mathrm{p}=0.054)(\mathrm{p}=0.048$ for LAVmin vs. LAVmax). LAVmin $\geq 23 \mathrm{ml} / \mathrm{m} 2$ was associated with LVEDP elevation with $86 \%$ sensitivity and $63 \%$ specificity. Of the LAEF indices global LAEF had the closest association with LVEDP elevation (AUC $0.780, \mathrm{p}<0.001$ ), followed by active LAEF (AUC 0.698, $\mathrm{p}=0.017$ ) and passive LAEF (AUC 0.646, $\mathrm{p}=0.099$ ). Global LAEF $\leq 35 \%$ was associated with LVEDP elevation with $71 \%$ sensitivity and $89 \%$ specificity while active LAEF $\leq 16 \%$ was associated with LVEDP elevation with $43 \%$ sensitivity and $93 \%$ specificity.

\section{Conclusions}

LAV measured at LV end diastole had a stronger association with LVEDP than LAV measured at LV end systole. Global and active LAEF were significantly reduced with increasing LVEDP.

\section{Funding}

None.

Published: 1 February 2012

doi:10.1186/1532-429X-14-S1-P233

Cite this article as: Posina et al:: Relationship of left atrial size and

function to invasive left ventricular filling pressure: a cardiac MRI study. Journal of Cardiovascular Magnetic Resonance 2012 14(Suppl 1):P233.

Saint Francis Hospital, Glen Cove, NY, USA

(c) 2012 Posina et al; licensee BioMed Central Ltd. This is an open access article distributed under the terms of the Creative Commons 